\title{
COMPORTAMENTO FÍSICO E AMBIENTAL DE RESÍDUOS SÓLIDOS EM PAVIMENTOS DE ESTRADAS FLORESTAIS ${ }^{1}$
}

\author{
Carlos Cardoso Machado², José Maurício Machado Pires ${ }^{3}$ e Reginaldo Sérgio Pereira ${ }^{4}$
}

\begin{abstract}
RESUMO - O objetivo deste estudo foi avaliar os parâmetros físicos de solos da região de Viçosa, Minas Gerais, Brasil, e misturas de rejeitos industriais conhecidos como lama-de-cal e grits. A caracterização foi realizada através dos ensaios de análise granulométrica, análise térmica e microscopia eletrônica de varredura. Foram selecionadas duas amostras de solo residual, ETA (argiloso) e VS (arenoso), as quais foram misturadas com $16,18,20,22$ e $24 \%$ de lama-de-cal e grits (em peso de matéria seca). As misturas de solo-lama-de-cal e solo-grits apresentaram grande capacidade de retenção de metais pesados, o que impossibilita a sua interação com o sistema hídrico pela possibilidade de contaminação do lençol freático e cursos d'água.
\end{abstract}

Palavras-chave: Análise granulométrica, análise térmica e microscopia eletrônica de varredura.

\section{PHYSICAL AND ENVIRONMENTAL BEHAVIOR OF SOLID WASTE IN FOREST ROAD PAVEMENTS}

\begin{abstract}
Abstract: The objective of the present study was the assessment of physical parameters of mature and young residual soils from the municipality of Viçosa, Minas Gerais, Brazil, as well as their mixtures with industrial waste known as whitewash mud and grits. Physical characterization was carried out through laboratory tests such as sieve analysis, thermal analysis and scanning microscopy. Two residual soil samples were chosen for this study, ETA (a clayey soil) and VS (a sandy soil), which were stabilized with $16 \%, 18 \%, 20 \%, 22 \%$ e $24 \%$ (referred to dry weight) of residual wastes aforementioned. Data from laboratory testing program supported that mixtures of soil and residual waste had a high heavy metal retention capacity, which might be of concern due to the possibility of contamination of water table and local streams.
\end{abstract}

Keywords: Sieve analysis, thermal analysis and scanning microscopy.

\section{INTRODUÇÃO}

Em estudos envolvendo solos, o conhecimento das suas características químicas e físicas é fundamental para melhor entendê-los. As características químicas são importantes no que diz respeito aos seus comportamentos em relação às suas trocas catiônicas e aniônicas em seus complexos sortivos (INGLES e METCALF, 1977). Dependendo de sua composição química, um solo poderá apresentar-se mais ou menos ativo, ou seja, as trocas de cátions ou de ânions poderão ocorrer com mais ou menos facilidade. Parâmetros físicos de um solo são indicadores do seu comportamento mecânico e estão relacionados às suas propriedades químicas (SINGH, 1967; MERLIM e RICHARD, 1982). Um solo constituído, por exemplo, de minerais de caulinita, goethita e gibsita irá trocar menos cátions do que um que contenha, por exemplo, minerais de vermiculita ou esmectita (SHERWOOD, 1961).

Assim, o objetivo deste trabalho foi avaliar as propriedades físicas de dois solos característicos da

\footnotetext{
${ }^{1}$ Recebido em 31.08.2006 e aceito para publicação em 29.03.2007

${ }_{2}^{2}$ Departamento de Engenharia Floresta da Universidade Federal de Viçosa (UFV). E-mail: <machado@ufv.br>.

${ }^{3}$ Doutorando de Engenharia Civil da UFV. E-mail: <machado.pires@ terra.com.br>.

${ }^{4}$ Departamento de Engenharia Florestal da Universidade de Brasília. E-mail: <reginaldosp@ unb.br>.
} 
microrregião de Viçosa, Zona da Mata Norte de Minas Gerais, e de suas misturas com os resíduos sólidos industriais lama-de-cal e grits, oriundos da indústria de celulose, com vistas ao entendimento do possível comportamento ambiental dessas misturas para o seu emprego em camadas de pavimentos de estradas florestais.

\section{MATERIAL E MÉTODOS}

\subsection{Solos e resíduos industriais}

Dois solos da microrregião de Viçosa, MG, foram empregados neste estudo. Uma das amostras designada por ETA foi coletadas no horizonte B de um talude de corte próximo à estação de tratamento de água de Viçosa, MG; trata-se de um Latossolo Vermelho-Amarelo, laterítico. A outra amostra designada por VS foi coletada no horizonte $\mathrm{C}$ de um talude de corte situado na localidade conhecida por Vila Secundino, no campus da Universidade Federal de Viçosa (UFV); trata-se de um solo de comportamento não-laterítico. Os resíduos sólidos industriais empregados foram a lama-de-cal e o grits. Esses materiais são provenientes de uma das etapas do processo kraft de extração de celulose, durante a recuperação da soda cáustica.

\subsection{Misturas realizadas}

Os ensaios foram realizados com as amostras de solo e as misturas solo-resíduo. Os teores de resíduos empregados nas misturas foram de 16, 18, 20, 22 e 24\%, calculados sobre a massa de solo seco. Esses teores foram escolhidos com base nos resultados de resistência mecânica obtidos por Pereira (2005), em estudos de misturas solo-grits e solo-lama de cal para aplicação em estradas florestais.

\subsection{Ensaios}

Os ensaios realizados com as amostras de solo e com as misturas solo-resíduo, bem como as respectivas normatizações técnicas, são descritos como se segue:

a) Análise granulométrica

Foi realizada pelo método da pipeta (dispersão total). Para tanto, todas as amostras foram tratadas com hexametafosfato de sódio, tamponada com carbonato de sódio anidro, devido à grande quantidade de sais presentes nos resíduos, segundo as recomendações da Embrapa (1997).

b) Análise de raios- $X$

Através do emprego da técnica de difratometria de raios-X foi possível a identificação qualitativa dos minerais presentes nos solos e nas misturas solo-resíduo. A metodologia empregada baseou-se nas recomendações da Embrapa (1997).

\section{c) Análise térmica}

Tomaram-se $100 \mathrm{mg}$ de amostras de solo e de misturas solo-resíduo para a obtenção do termograma de Análise Termodiferencial (ATD). No equipamento, as amostras foram submetidas a um aquecimento de $10{ }^{\circ} \mathrm{C}$ por minuto em atmosfera de nitrogênio, segundo a metodologia proposta por Mackenzie (1956).

d) Análises de microscopia eletrônica de varredura (MEV)

A técnica de microscopia eletrônica de varredura foi empregada para a determinação da morfologia dos minerais contidos nos solos e nas misturas solo-resíduo. Para tanto, uma fita de carbono foi usada para fixar o material analisado no microscópio eletrônico de varredura. O emprego da fita de carbono foi para evitar que partículas caiam na câmara de ionização e causem danos físicos ao MEV.

\section{RESULTADOS E DISCUSSÃO}

\subsection{Análises granulométricas dos solos e das misturas solo-resíduo}

No Quadro 1, apresentam-se os resultados das análises granulométricas das amostras de solo em seus estados naturais e em misturas com os resíduos lama-de cal e grits em diferentes concentrações. Machado e Pires et al. (2004) observaram que a utilização convencional de solução de $\mathrm{NaOH}$ como dispersante teve de ser substituída pelo hexametafosfato de sódio por causa da alta concentração de cátions no sistema que promovia a floculação em vez da dispersão da fração argila.

\subsection{Análises de Raios- $X$}

O Quadro 2 contém os resultados das análises por difração de raios-X das amostras de solo e das misturas solo-resíduo. De modo geral, encontraramse caulinita (C), gibsita (G), goethita (Go) e quartzo (Q). A Figura 1 ilustra os difratogramas das misturas solo $+22 \%$ lama-de-cal e solo $+22 \%$ grits. Pires e Machado et al. (2003) observaram que essa técnica em conjunto com a análise térmica indicou que minerais de fase hidratada, minerais com água de cristalização em sua estrutura química, promovem a retenção de metais pesados nos seus retículos cristalinos, evitando que esses metais se percolem no sistema hídrico. 
Quadro 1 - Granulometria dos solos e das misturas solo-resíduo Table 1 -Sieve analysis of the soils and soil-waste mixtures

\begin{tabular}{|c|c|c|c|c|c|}
\hline \multirow[t]{2}{*}{ Amostras } & \multicolumn{2}{|c|}{$\%$ Areia } & \multirow[t]{2}{*}{$\%$ Silte } & \multirow[t]{2}{*}{$\%$ Argila } & \multirow[t]{2}{*}{ Classe Textural } \\
\hline & Grossa & Fina & & & \\
\hline ETA natural & 6 & 7 & 8 & 79 & Muito argilosa \\
\hline ETA com $16 \%$ lama de cal & 6 & 8 & 13 & 73 & Muito argilosa \\
\hline ETA com $18 \%$ lama de cal & 5 & 6 & 11 & 78 & Muito argilosa \\
\hline ETA com $20 \%$ lama de cal & 5 & 6 & 11 & 78 & Muito argilosa \\
\hline ETA com $22 \%$ lama de cal & 5 & 6 & 10 & 79 & Muito argilosa \\
\hline ETA com $24 \%$ lama de cal & 7 & 6 & 10 & 77 & Muito argilosa \\
\hline ETA com $16 \%$ grits & 9 & 10 & 23 & 58 & Argilosa \\
\hline ETA com $18 \%$ grits & 6 & 8 & 27 & 59 & Argilosa \\
\hline ETA com $20 \%$ grits & 5 & 6 & 31 & 58 & Argilosa \\
\hline ETA com $22 \%$ grits & 5 & 6 & 29 & 56 & Argilosa \\
\hline ETA com $24 \%$ grits & 5 & 6 & 28 & 55 & Argilosa \\
\hline VS natural & 42 & 27 & 23 & 8 & Franco arenosa \\
\hline VS com $16 \%$ lama de cal & 40 & 27 & 23 & 10 & Franco arenosa \\
\hline VS com $18 \%$ lama de cal & 42 & 25 & 23 & 10 & Franco arenosa \\
\hline VS com $20 \%$ lama de cal & 40 & 29 & 23 & 8 & Franco arenosa \\
\hline VS com $22 \%$ lama de cal & 42 & 29 & 25 & 8 & Franco arenosa \\
\hline VS com $24 \%$ lama de cal & 44 & 25 & 23 & 8 & Franco arenosa \\
\hline VS com $16 \%$ grits & 40 & 27 & 23 & 10 & Franco arenosa \\
\hline VS com $18 \%$ grits & 42 & 25 & 23 & 10 & Franco arenosa \\
\hline VS com $20 \%$ grits & 40 & 29 & 23 & 8 & Franco arenosa \\
\hline VS com $22 \%$ grits & 42 & 29 & 25 & 8 & Franco arenosa \\
\hline VS com $24 \%$ grits & 44 & 25 & 23 & 8 & Franco arenosa \\
\hline
\end{tabular}

Quadro 2 - Análise por difração de raios X dos solos e das misturas solo-resíduo

Table 2 -X-ray diffraction analysis of soils and soil-waste mixtures

\begin{tabular}{lc}
\hline \multicolumn{1}{c}{ Amostras } & Mineralogia \\
\hline ETA natural & $\mathrm{C} ; \mathrm{Q} ; \mathrm{Gb}$ \\
ETA com 16\% lama de cal & $\mathrm{C} ; \mathrm{G} ; \mathrm{Go}$ \\
ETA com 18\% lama de cal & $\mathrm{C} ; \mathrm{G} ; \mathrm{Go}$ \\
ETA com 20\% lama de cal & $\mathrm{C} ; \mathrm{G} ; \mathrm{Go}$ \\
ETA com 22\% lama de cal & $\mathrm{C} ; \mathrm{G} ; \mathrm{Go}$ \\
ETA com 24\% lama de cal & $\mathrm{C}$ (desloc.); G; Go \\
ETA com 16\% grits & $\mathrm{C}, \mathrm{Q}, \mathrm{Gb}$ \\
ETA com 18\% grits & $\mathrm{C}, \mathrm{Q}, \mathrm{Gb}$ \\
ETA com 20\% grits & $\mathrm{C}, \mathrm{Q}, \mathrm{Gb}$ \\
ETA com 22\% grits & $\mathrm{C}, \mathrm{Q}, \mathrm{Gb}$ \\
ETA com 24\% grits & $\mathrm{C}, \mathrm{Q}, \mathrm{Gb}$ \\
VS natural & $\mathrm{M}, \mathrm{C}, \mathrm{Q}, \mathrm{Gb}$ \\
VS com 16\% lama de cal & $\mathrm{C} ; \mathrm{G}$ \\
VS com 18\% lama de cal & $\mathrm{C} ; \mathrm{G} ; \mathrm{Q}$ \\
VS com 20\% lama de cal & $\mathrm{C} ; \mathrm{G} ; \mathrm{Q}$ \\
VS com 22\% lama de cal & $\mathrm{C} ; \mathrm{G} ; \mathrm{Q}$ \\
VS com 24\% lama de cal & $\mathrm{C} ; \mathrm{Q} ; \mathrm{G}$ \\
VS com 16\% grits & $\mathrm{M}, \mathrm{C}, \mathrm{Q}, \mathrm{Gb}$ \\
VS com $18 \%$ grits & $\mathrm{M}, \mathrm{C}, \mathrm{Q}, \mathrm{Gb}$ \\
VS com 20\% grits & $\mathrm{M}, \mathrm{C}, \mathrm{Q}, \mathrm{Gb}$ \\
VS com 22\% grits & $\mathrm{M}, \mathrm{C}, \mathrm{Q}, \mathrm{Gb}$ \\
VS com 24\% grits & $\mathrm{M}, \mathrm{C}, \mathrm{Q}, \mathrm{Gb}$ \\
\hline
\end{tabular}

\subsection{Análises Térmicas}

A Figura 2 ilustra os termogramas das amostras das misturas de solo ETA e VS com os resíduos sólidos lama-de-cal e grits.

\subsection{Análises da Microscopia Eletrônica de Varredura (MEV)}

As micrografias do MEV dos resultados das misturas solo-resíduo encontram-se na Figura 3. Essa técnica é muito utilizada para verificar morfologia de solos e suas interações com os resíduos sólidos industriais lama de cal e grits. A morfologia do solo ETA com a lama-de-cal apresentou uma diferenciação dos componentes da fração argila do solo com a lama de cal, já na mistura do solo VS com a lama-de-cal houve certo "mascaramento", ou seja, não foi possível diferenciar, porque esse solo é muito arenoso, e isso dificultou distinguir o solo do resíduo. 
a) Mistura solo ETA + $22 \%$ Lama de Cal

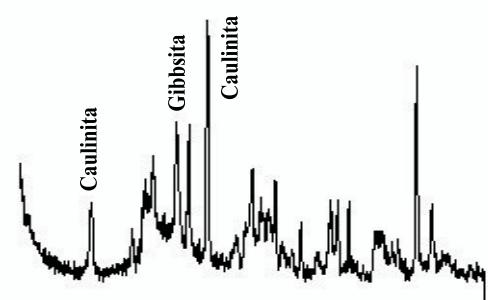

b) Mistura solo ETA $+22 \%$ Grits
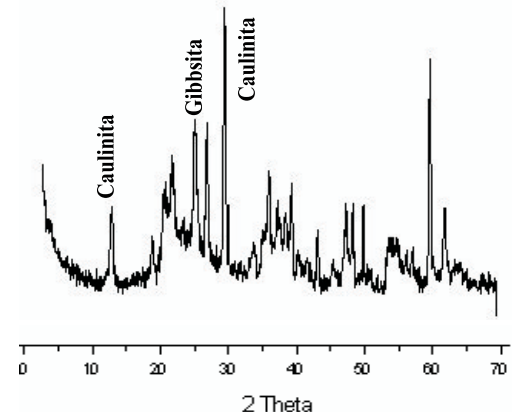

c) Mistura solo VS + $22 \%$ Lama de Cal

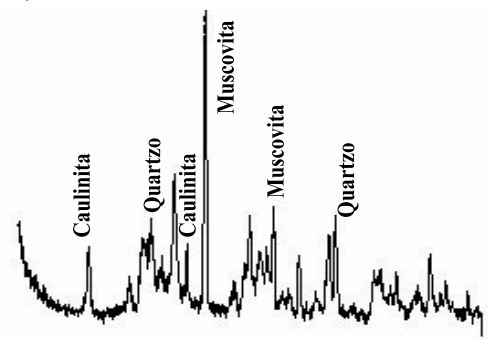

d) Mistura solo VS $+22 \%$ Grits
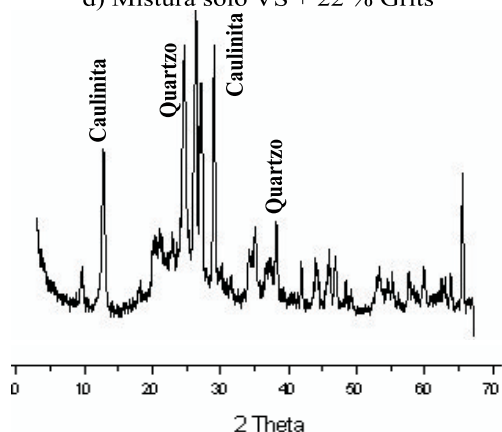

Figura 1 - Difratogramas das misturas solo $+22 \%$ de resíduo.

Figure 1-Diffratogram of the soil $+22 \%$ waste mixtures.
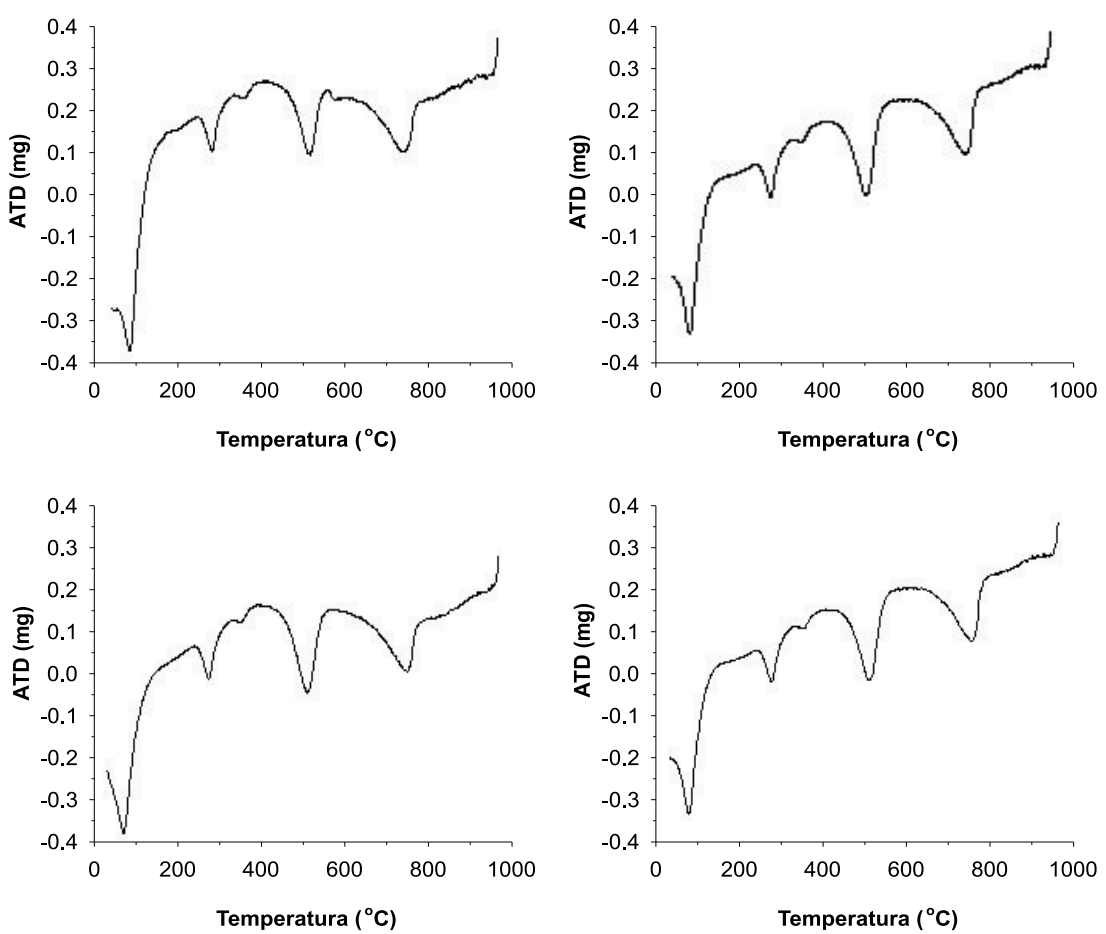

Figura 2 - Análise térmica das misturas solo + 22\% resíduo: (a) ETA + lama de cal; (b) VS + lama de cal; (c) ETA + grits; e (d) VS + grits.

Figure 2 - Thermal analysis of the soil $+22 \%$ waste mixtures: (a) ETA + whitewash mud; mixtures $(b)$ VS + whitewash mud mixtures; (c) ETA + grits mixtures and (d) VS + grits mixtures.

R. Árvore, Viçosa-MG, v.31, n.4, p.679-684, 2007 


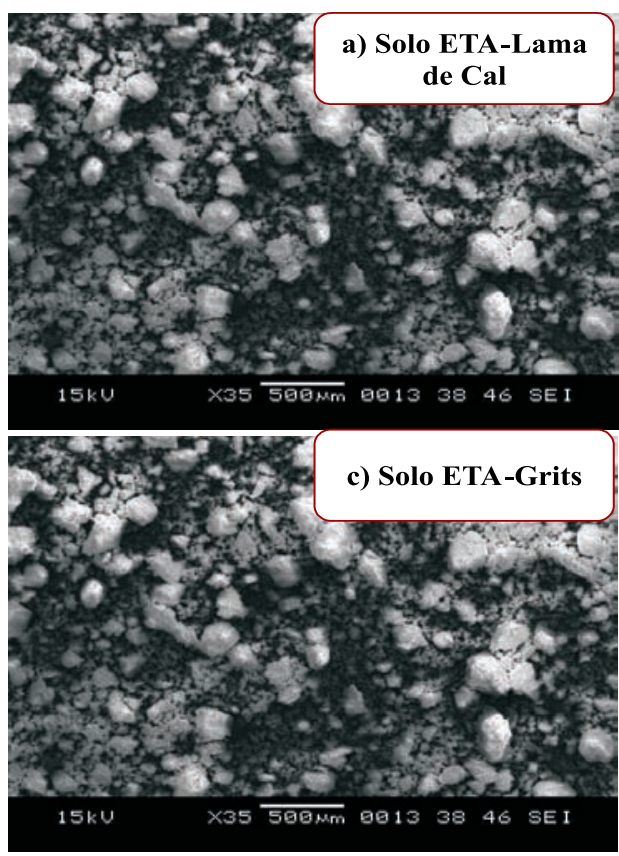

Figura 3 - Micrografias de misturas solo $+22 \%$ resíduo. Figure 3-Micrography of the soil $+22 \%$ waste mixtures.

\section{CONCLUSÕES}

As seguintes conclusões foram extraídas do trabalho: (i) o teor de caulinita e de goethita está na faixa de 20 a $50 \%$, significando que esses solos naturais e as misturas solo-resíduo apresentam grande capacidade de retenção de metais pesados, impossibilitando-os de disponibilizar para o sistema hídrico; e (ii) os solos analisados possuem baixo teor de alumínio advindo do argilomineral caulinita, impedindo a ocorrência de reações pozolânicas com a lama-de-cal.

\section{REFERÊNCIAS}

\section{ASSOCIAÇÃO BRASILEIRA DE NORMAS} TÉCNICAS - ABNT. NBR 10.004: classificação de resíduos sólidos. Rio de Janeiro: 1987. 63p.

\section{ASSOCIAÇÃO BRASILEIRA DE NORMAS} TÉCNICAS - ABNT. NBR 10.005: lixiviação de resíduos sólidos. Rio de Janeiro: 1987.7p.

\section{ASSOCIAÇÃO BRASILEIRA DE NORMAS} TÉCNICAS - ABNT. NBR 10.006:

solubilização de resíduos sólidos. Rio de Janeiro: 1987. 2p.

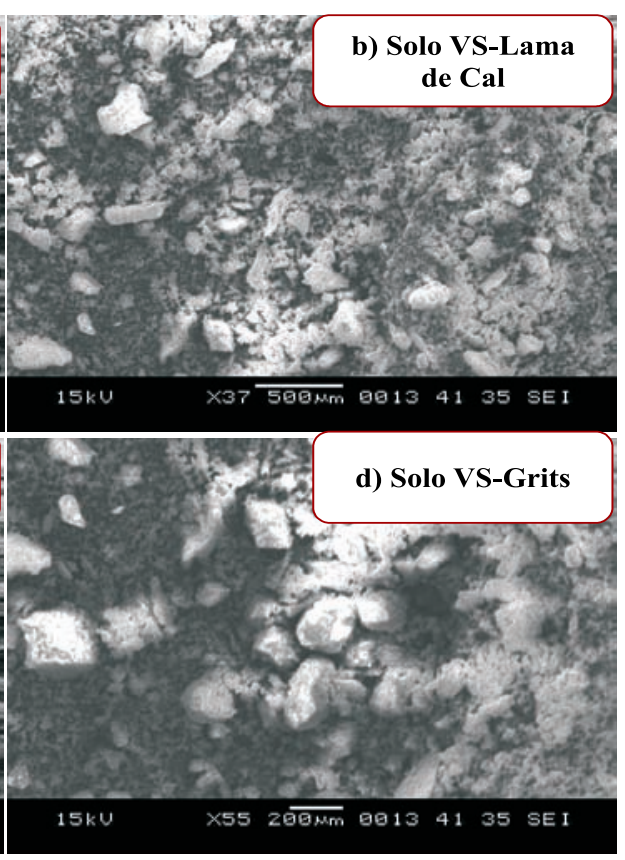

ASSOCIAÇÃO BRASILEIRA DE NORMAS TÉCNICAS - ABNT. NBR 10.007: amostragem de resíduos sólidos. Rio de Janeiro: 1987. 25p.

INGLES, O. G.; METCALF, J. B. Soil stabilization principles and practice. New York: Haested Press, 1977. 374p.

MACHADO, C. C.; PEREIRA, R. S.; PIRES, J. M. M. Influência do tratamento térmico do resíduo sólido industrial (grits) na resistência mecânica de um latossolo para pavimentos de estradas florestais. Revista Árvore, v.27, n.4, p.543-550, 2003.

MACHADO, C. C. et al. Classificação tecnológica de solos e resíduos industriais, com e sem tratamento térmico, para fins rodoviários. Revista Árvore, v.27, n.5, p.657-668, 2003.

MACHADO, C. C. et al. Environmental behavior of soils and mixtures of soil - whitewash-mud. Revista Árvore, v.28, n.3, p.459-464, 2004.

R. Árvore, Viçosa-MG, v.31, n.4, p.679-684, 2007 
MACHADO, C. C. et al. Utilização do resíduo sólido (lama de cal) em pavimentos de estradas florestais. Revista Árvore, v.28, n.4, p.547-551, 2004.

MACKENZIE, R. C. et al. The thermal methods. Aberdeen: The Macaulay Institute for Soil Research, 1956. 456p.

MERLIM, G. S.; RICHARD, L. H. Soil

engineering. 4.ed. São Paulo: Harper \& Row Publishers, 1982. 819p.
PIRES, J. M. M. et al. Potencial poluidor de resíduo sólido da samarco mineração: estudo de caso da barragem de germano. Revista Árvore, v.27, n.3, p.393-397, 2003.

SHERWOOD, P. T. Soil stabilization by the use of chemical admixtures. roads and road constructions S.L.: 1961. p.102-110.

SINGH, A. Soil engineering in theory and practice. London: Asia Publishing House, 1967. 653p. 